\title{
The prognostic significance of the mucin phenotype of gastric adenocarcinoma and its relationship with histologic classifications
}

\author{
OK-JAE LEE ${ }^{1}$, HYUN-JIN KIM ${ }^{1}$, JANG-RAK KIM ${ }^{2}$ and HIDENOBU WATANABE ${ }^{3}$ \\ ${ }^{1}$ Department of Internal Medicine, Institute of Health Science; ${ }^{2}$ Department of Preventive \\ Medicine and Biostatistics, Gyeongsang National University School of Medicine, Jinju, Korea; \\ ${ }^{3}$ First Department of Pathology, Niigata University School of Medicine, Niigata, Japan
}

Received September 22, 2008; Accepted November 27, 2008

DOI: $10.3892 /$ or_00000234

\begin{abstract}
The prognostic value of histologic classifications of gastric adenocarcinoma is controversial, although they have been commonly used. The clinical significance of the mucin phenotype has not been clarified. This study was conducted to determine the clinical significance of mucin phenotype as a possible prognostic factor. Mucin histochemistry by paradoxical concanavalin A (Con A) staining and immunostaining for $45 \mathrm{M} 1, \mathrm{MUC} 2$ glycoprotein and CD10 of mucin was performed in surgically obtained paraffin-embedded specimens from 106 gastric adenocarcinomas. We determined their mucin phenotypes and analyzed their relationships with clinical and histopathologic variables and survival rates. Among 106 gastric adenocarcinomas, $37(34.9 \%), 35(33.0 \%), 22(20.8 \%)$ and 12 $(11.3 \%)$ expressed the intestinal (I-), the gastric (G-), mixed (M-), and undetermined (U-) phenotypes, respectively. Although the mucin phenotype correlated well with histologic differentiation $(\mathrm{p}=0.000)$ and Lauren's classification of a tumor $(\mathrm{p}=0.003)$, it did not accord completely with them. There was no relationship between mucin phenotype and other patient clinicopathologic variables. No statistically significant difference in survival was observed among mucin phenotypes on univariate $(\mathrm{p}=0.089)$ and multivariate $(p=0.088)$ analyses. However, the patients with I-phenotype tumor had a significantly better outcome than those with nonI-phenotype tumor on univariate $(\mathrm{p}=0.023)$ and multivariate $(\mathrm{p}=0.049)$ analyses. In conclusion, the mucin phenotype did not accord completely with histologic differentiation and Lauren's classification of gastric adenocarcinoma, despite a well-defined correlation between them. I-phenotypic expression, but not the histologic differentiation and Lauren's
\end{abstract}

Correspondence to: Dr Ok-Jae Lee, Department of Internal Medicine, Gyeongsang National University School of Medicine, 90 Chilam-dong, Jinju, Gyeongnam 660-702, Korea E-mail: ojlee@gnu.ac.kr

Key words: gastric cancer, mucin phenotype, prognosis, Lauren's classification, differentiation classification, was found to be an independent good prognostic factor of gastric cancers.

\section{Introduction}

Gastric adenocarcinoma is histologically classified as intestinal vs. diffuse, or differentiated vs. undifferentiated, based on the tendency of gland formation $(1,2)$. The prognostic value of these histologic classifications of gastric adenocarcinoma is controversial, although they have been commonly used $(3,4)$.

Recent advances in mucin histochemistry and immunohistochemistry have demonstrated that gastric and intestinal phenotypic cell markers are widely expressed in gastric carcinomas, irrespective of their histologic type (5-9). The mucin expression pattern of gastric carcinomas is heterogeneous. Decreased expression of gastric mucins and de novo expressions of MUC2 have also been described in intestinal metaplasia, a precancerous lesion of gastric carcinomas. These findings suggest that the repertoire of mucins synthesized by gastric cancer cells is tightly associated with their differentiation program. Therefore, the pattern of mucin expression may provide new insights into the differentiation pathways of gastric cancers (10-14).

Specific antibodies to the various kinds of mucins can be used to define mucin phenotypes of gastric adenocarcinomas. Several authors have reported that gastric carcinomas can be classified as having either a gastric (G-), gastric and intestinal mixed (M-) or intestinal (I-) phenotype, depending on mucin histochemistry with Con A and immunopositivity of HGM, MUC6, MUC2 and CD10 stainings (7-9). The phenotypic marker expression of tumors is conventionally thought to imitate that of the tissue of origin. Thus, previous data suggest that gastric carcinomas may occur in various types of gastric mucosa, although differentiated tumors have generally arisen from gastric mucosa with intestinal metaplasia, while undifferentiated tumors have arisen from ordinary gastric mucosa without intestinal metaplasia $(1,2)$. Tajima et al (9) previously reported that G-phenotype tumors accounted for $27.7 \%$ of differentiated tumors, often referred to as intestinal-type tumors by Lauren (1), while I-phenotype tumors accounted for $10.1 \%$ of undifferentiated tumors. These findings raise the question of whether the expression of mucin phenotypes depends on the differentiation pattern of 
carcinomas. However, no sufficient data are available concerning the association of certain histologic subtypes with the expression of these antigens. Additionally, although recent results have observed that G-phenotype tumors are associated with a poorer outcome and a greater potential for malignancy compared to other phenotypes, the relationship between a mucin phenotypic expression and the prognosis of gastric carcinomas has not been established (6-9).

Hence, we investigated the mucin phenotypic expression of gastric adenocarcinomas based on histochemistry and immunohistochemistry in regard to Con A binding and 45M1, MUC2 and CD10 antigens. In addition, mucin phenotypic expression was correlated to various clinical and histopathologic variables as well as overall survival to clarify the difference from conventional histologic differentiation and to evaluate its prognostic significance.

\section{Materials and methods}

Patient samples. This study was based on the analysis of 106 surgically resected primary gastric adenocarcinomas. All tumor tissues were collected randomly during June, 1998 through December, 2002 at Gyeongsang National University Hospital. Tissue samples and clinical and histopathologic information for each case were obtained according to approved institutional guidelines. Informed consent was obtained from all 106 patients with gastric adenocarcinomas. The clinical and histopathologic data of all patients are summarized in Table I. Seventy-two patients were male with a mean age of 57.8 years (range: 26-78 years). The infection rate of Helicobacter pylori (H. pylori) was $89.7 \%$ (70/78). Most gastric cancers were located in the non-cardia region (antrum, 44; body, 41; antrum and body, 17; cardia, 4). Forty patients had well-differentiated cancers and 66 had poorly differentiated cancers. According to Lauren's classification, 44 lesions were intestinal type and 62 were diffuse type. The TNM stage of the gastric cancers was 1:2:3:4 in 36:26:24:20. The histologic classification was based on Lauren's classification (1) and the WHO classification (15), where papillary and well- to moderately-differentiated tubular adenocarcinomas were considered well-differentiated cancers, while poorly differentiated tubular, signet ring cells and mucinous type adenocarcinomas were classified as poorly differentiated.

Patients who had undergone chemo- or radiotherapy prior to surgery and had a past medical history of gastric cancer were excluded from the study. After resection, the surgical specimens were fixed in $10 \%$ buffered-formalin, embedded in paraffin wax, and examined carefully at the macroscopic level. All sections were routinely prepared and stained with hematoxylin and eosin ( $H \& E)$. After examining the $H \& E$ sections, the tumor section that had the most well-preserved mucosal components was selected as a representative sample. Paraffin blocks corresponding to the selected H\&E sections were cut into $5-\mu \mathrm{m}$ consecutive sections for histochemical and immunohistochemical staining. Infection by $H$. pylori was detected by the rapid urease test (RUT) and histologic examination and/or serology. Seven or eight biopsy samples were obtained from the grossly non-neoplastic area of each resected stomach and used for RUT. Two biopsy samples
Table I. Clinicopathologic features of 106 patients with gastric adenocarcinoma.

\begin{tabular}{lc}
\hline Clinical parameter & $\begin{array}{c}\text { No. of patients } \\
(\%)\end{array}$ \\
\hline Age (years) & \\
Mean (range) & $57.8(26-78)$ \\
Gender & \\
Male & $72(67.9)$ \\
Female & $34(32.1)$ \\
H.pylori infection & $70 / 78(89.7)$ \\
Location of tumor & \\
Antrum & $44(41.5)$ \\
Body & $41(38.7)$ \\
Antrum and body & $17(16.0)$ \\
Cardia & $4(3.8)$ \\
Histologic differentiation & \\
Well-differentiated & $40(37.7)$ \\
Poorly-differentiated & $66(62.3)$ \\
Lauren's classification & \\
Intestinal type & \\
Diffuse type & $44(41.5)$ \\
Stage & $62(58.5)$ \\
I & \\
II & $36(34.0)$ \\
IV & $26(24.5)$ \\
\hline
\end{tabular}

were obtained from the antrum and corpus, respectively, during endoscopy and examined microscopically to detect H. pylori. A histologic interpretation of the gastric biopsies was performed by pathologists who are experts at identifying $H$. pylori. If more than one test was positive, the case was regarded as positive for $H$. pylori. If all tests were negative, the case was regarded as negative.

Antibodies for mucin histochemistry and immunohistochemistry. To determine the mucin phenotypes of the gastric adenocarcinomas, a combination of human gastric mucin (45M1), Con A, MUC2, and CD10 staining was carried out. For identification of pyloric gland-type mucin, histochemical staining with paradoxical concanavalin A class III (Con A, Kanto Chemical Co., Tokyo, Japan) was performed according to the method of Katsuyama and Spicer (16). The paradoxical Con A staining method (PCS: periodate oxidation, borohydrate reduction, and Con A staining) is a variant of the Con A peroxidase method.

For immunohistochemistry, the primary antibodies against human gastric mucin antibody NCL-HGM-45M1 (Novocastra, Newcastle, UK; diluted 1:50; stains mucin of the surface gastric epithelium), MUC2 (Novocastra; diluted 1:500; stains intestinal mucin), and CD10 (Novocastra; diluted 1:200; stains brush border of gut epithelial cells) were used. 


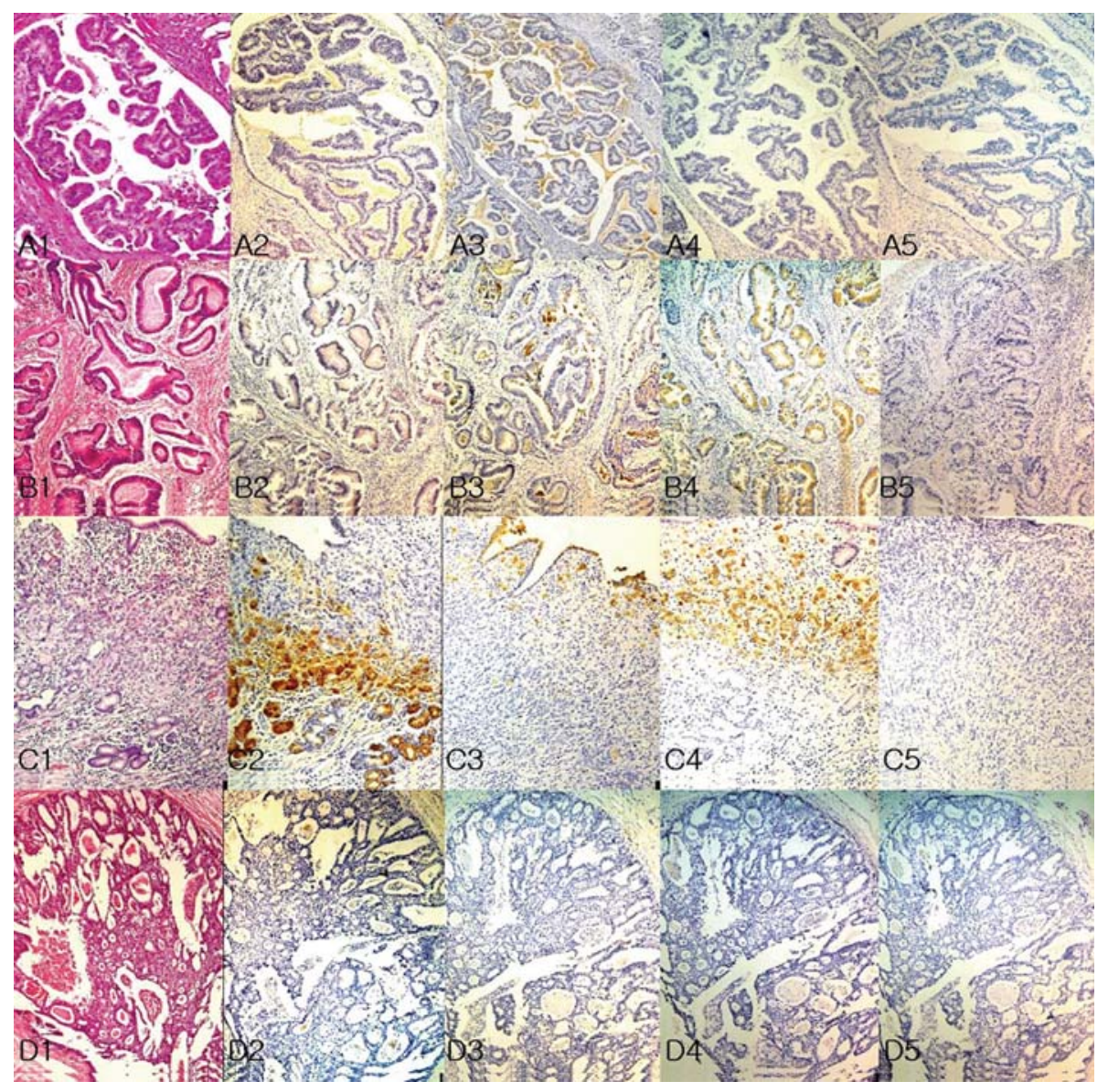

Figure 1. Histochemical and immunohistochemical analyses for mucin glycoproteins. (A) Gastric adenocarcinoma expressing gastric phenotype. Welldifferentiated tubular adenocarcinoma, Lauren's intestinal type (A1, H\&E; original magnification, x100). The tumor cells are negative for paradoxical concanavalin A (A2, Con A; original magnification, x100) but positive for 45M1 (A3, 45M1; original magnification, x100). The tumor cells are negative for MUC2 (A4, MUC2; original magnification, x100) and CD10 (A5, CD10; original magnification, x100). (B) Gastric adenocarcinoma expressing intestinal phenotype. Well-differentiated tubular adenocarcinoma, Lauren's intestinal type (B1, H\&E; original magnification, x100). The tumor cells are negative for paradoxical concanavalin A (B2, Con A; original magnification, x100). A few cancer cells ( $<5 \%)$ and trapped normal epithelial cells are positive for 45M1 (B3, 45M1; original magnification, x100). The tumor cells are negative for MUC2 and CD10 (B4, MUC2; original magnification, x100; B5, CD10; original magnification, x100). (C) Gastric adenocarcinoma expressing mixed phenotype. Signet ring cell carcinoma, Lauren's diffuse type (C1, H\&E; original magnification, x100). Cancer cells are positive for concanavalin A (C2, Con A; original magnification, x100), 45M1 (C3, 45M1; original magnification, x100) and MUC2 (C4, MUC2; original magnification, x100), but negative for CD10 (C5, CD10; original magnification, x100). (D) Gastric adenocarcinoma expressing unclassified phenotype. Well-differentiated tubular adenocarcinoma, Lauren's intestinal type (D1, H\&E; original magnification, x100). Cancer cells are negative for concanavalin A (D2, Con A; original magnification, x100), 45M1 (D3, 45M1; original magnification, x100), MUC2 (D4, MUC2; original magnification, x100), and CD10 (D5, CD10; original magnification, x100).

The immunohistochemical staining procedure. Paraffinembedded tissue blocks were cut into $5-\mu \mathrm{m}$ sections. After deparaffinizing with xylene and rehydrating with descending concentrations of ethanol, antigen retrieval was performed by placing slides in citrate buffer ( $\mathrm{pH} 7.0$ ) in a microwave oven at $300 \mathrm{~W}$ for $20 \mathrm{~min}$. The sections were incubated with each primary antibody, 45M1, MUC2, or CD10. Staining procedures using a Dako LSAB kit (Dako, Carpinteria, CA, USA) were performed according to the manufacturer's instructions.

Sections were then counterstained with hematoxylin, mounted, and evaluated by two experienced pathologists. In some cases where the two evaluations led to different results, a consensus interpretation was reached after re-examination. We used non-neoplastic gastric mucosa as an internal control for mucin immunohistochemical staining. The specificity of immunostaining was checked by omitting single steps in the protocol and by replacing the primary antibody with a nonimmune serum.
Classification of mucin phenotypes of adenocarcinomas. The mucin phenotypes of gastric cancers were classified into four categories according to the pattern of immunostaining for CD10, MUC2, HGM and lectin staining with Con A: gastric type (G-phenotype); intestinal type (I-phenotype); gastrointestinal mixed type (M-phenotype); and unclassified type (U-phenotype, no mucin reactive cells). The G-phenotype cancer was defined as a tumor with predominantly intracytoplasmic mucin of the gastric phenotype, as determined by immunostaining of human gastric mucin (45M1) and/or of pyloric gland mucin (Con A), but without MUC2-positive intestinal-type cells or CD10-positive cells (Fig. 1A). The I-phenotype cancer referred to a tumor with MUC2-positive cells and/or CD10-positive cells (along with a brush border), but without Con A- or 45M1- positive gastric-type cells (Fig. 1B). M-phenotype cancer was defined as a tumor with immunostaining that was consistent with both G- and Iphenotypes (Fig. 1C). Finally, tumors without positive 

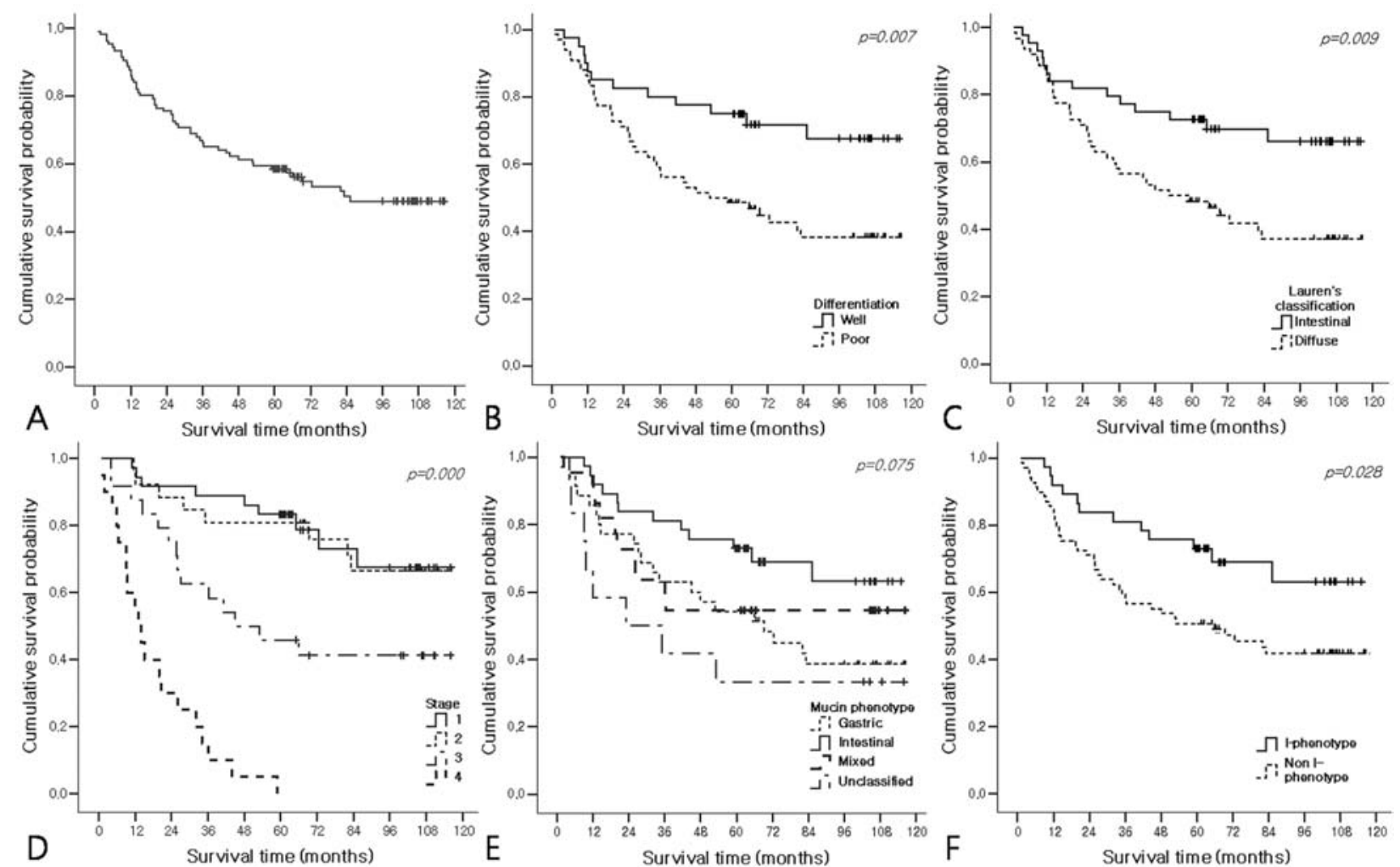

Figure 2. Cumulative 10-year survival curves according to histologic differentiation, Lauren's classification, tumor stages, and the mucin phenotypes. Overall 10 -year survival rate of patients with gastric adenocarcinoma is $51.9 \%$ and 5 -year survival rate is $56.6 \%$ (A). The survival rate is higher in welldifferentiated than poorly differentiated $(\mathrm{p}=0.013, \mathrm{~B})$, and in lower tumor stage than advanced stage $(\mathrm{p}=0.000, \mathrm{D})$. Lauren's classification and the mucin phenotype do not show a significant difference in survival ( $\mathrm{p}=0.073, \mathrm{C}$ and $\mathrm{p}=0.075, \mathrm{E})$ ( $\mathrm{p}$-values by log-rank test).

immunostaining consistent with G- or I-phenotypes were referred to as having a U-phenotype (Fig. 1D) (7-9).

If $>5 \%$ of the tumor cells were immunopositive, the tumor was regarded as positive. The distinct staining in $<5 \%$ of carcinoma cells was designated as negative, according to previous reports (17). However, if tumor cells were intensely stained with $\mathrm{CD} 10$, it was recorded as positive for CD10, regardless of the percentage (18).

Statistical analysis. Statistical calculations were performed using the SPSS 13.0 software package (SPSS Inc., Chicago, IL, USA). The correlation between mucin phenotypes and clinicopathologic variables was analyzed using the Pearson $\chi^{2}$ test. The overall survival curve was plotted using the KaplanMeier product-limit method. The relationship between survival and mucin phenotypes or clinicopathologic variables was analyzed using the log-rank test. Additionally, the survival rate was adjusted for the patient's age and gender, histologic differentiation, tumor stage, and Lauren's classification using the Cox proportional hazards model. $\mathrm{p}<0.05$ was considered statistically significant.

\section{Results}

The distribution of mucin phenotypes. Among 106 gastric adenocarcinomas, 35 (33.0\%) expressed the G-phenotype, 37 (34.9\%) the I-phenotype, 22 (20.8\%) the M-phenotype, and $12(11.3 \%)$ the U-phenotype (Table II).
Positive staining with respect to Con A, 45M1, MUC2, and CD10 was observed in 28 (26.4\%), $73(68.9 \%), 40$ $(37.7 \%)$, and $29(27.4 \%)$ lesions among 106 tumors, respectively (data not shown).

The relationship between mucin phenotype and clinicohistopathologic variables. There was no relationship between mucin phenotypic expression and patient's gender $(\mathrm{p}=0.172$, data not shown), tumor location $(\mathrm{p}=0.437$, data not shown), tumor stage ( $\mathrm{p}=0.296$, data not shown), presence of lymph node invasion or distant metastasis $(\mathrm{p}=0.151$ and 0.938 , respectively, data not shown), and $H$. pylori infection. $H$. pylori infection rates were $87.5,95.0,86.7$ and $90.9 \%$ in G-, I-, M- and U-phenotype cancers, respectively ( $\mathrm{p}=0.814)$. The depth of invasion was not either related to the mucin phenotype. However, I-phenotype was prominent in the early cancers, while G-phenotype was prominent in the advanced cancers $(\mathrm{p}=0.104)$. Among 26 early gastric cancers, G-, I- Mand $U$-phenotypes were 19.2, 57.7, 23.1 and 0.0\%, respectively. In 80 advanced gastric cancers, G-, I- M- and Uphenotypes accounted for 37.5, 27.5, 20.0 and 15.0\%, respectively. Among 14 differentiated types of early gastric cancers, $12(85.7 \%)$ were I-phenotypes, and 2 were Gphenotypes, respectively. There was no difference in the patients' age among the mucin types $(\mathrm{p}=0.123)$. The patients with I-phenotype tumors were older than those with non Iphenotype, but this difference did not reach statistical significance ( 60.5 vs. 56.3 years, $\mathrm{p}=0.082$ ). 
Table II. The relationship between mucin phenotype and histologic differentiation and Lauren's classification of gastric adenocarcinomas.

\begin{tabular}{|c|c|c|c|c|}
\hline \multirow[b]{2}{*}{ Mucin phenotype } & \multicolumn{2}{|c|}{ Histologic differentiation $(\%)^{\mathrm{a}}$} & \multicolumn{2}{|c|}{ Lauren's classification $(\%)^{\mathrm{b}}$} \\
\hline & Well & Poor & Intestinal & Diffuse \\
\hline Gastric $(n=35)$ & $7(20.0 / 17.5)$ & $28(80.0 / 42.4)$ & $10(28.6 / 22.7)$ & $25(71.4 / 40.3)$ \\
\hline Intestinal $(n=37)$ & $24(64.9 / 60.0)$ & $13(35.1 / 19.7)$ & $24(64.9 / 54.5)$ & $13(35.1 / 21.0)$ \\
\hline Mixed $(n=22)$ & $4(18.2 / 10.0)$ & $18(81.8 / 27.3)$ & $5(22.7 / 11.4)$ & $17(77.3 / 27.4)$ \\
\hline Unclassified $(\mathrm{n}=12)$ & $5(41.7 / 12.5)$ & $7(58.3 / 10.6)$ & $5(41.7 / 11.4)$ & $7(58.3 / 11.3)$ \\
\hline Total $(n=106)$ & $40(37.7 / 100)$ & $66(62.3 / 100)$ & $44(41.5 / 100)$ & $62(58.5 / 100)$ \\
\hline
\end{tabular}

${ }^{\mathrm{a}} \mathrm{p}=0.000$ by Pearson $\chi^{2}$ test; ${ }^{\mathrm{b}} \mathrm{p}=0.003$ by Pearson $\chi^{2}$ test.

Table III. Unadjusted and adjusted hazard ratios for death according to clinicopathologic variables including mucin phenotypes.

\begin{tabular}{|c|c|c|c|c|}
\hline \multirow{2}{*}{$\begin{array}{l}\text { Variable } \\
\text { (No. of patients) }\end{array}$} & \multicolumn{2}{|c|}{ Unadjusted analysis } & \multicolumn{2}{|c|}{ Adjusted analysis ${ }^{\mathrm{a}}$} \\
\hline & Hazard ratio $(95 \% \mathrm{CI})$ & p-value & Hazard ratio $(95 \% \mathrm{CI})$ & p-value \\
\hline Age & $1.01(0.99-1.04)$ & 0.299 & $1.05(1.02-1.07)$ & 0.002 \\
\hline \multicolumn{5}{|l|}{ Gender } \\
\hline Male (72) & 1.0 & 0.171 & 1.0 & 0.344 \\
\hline Female (34) & $0.64(0.34-1.21)$ & & $0.72(0.37-1.41)$ & \\
\hline Differentiation & & 0.009 & & 0.975 \\
\hline Well (40) & 1.0 & & 1.0 & \\
\hline Poor (66) & $2.36(1.24-4.52)$ & & $1.03(0.20-5.17)$ & \\
\hline Lauren's classification & & 0.011 & & 0.454 \\
\hline Intestinal (44) & 1.0 & & 1.0 & \\
\hline Diffuse (62) & $2.24(1.21-4.14)$ & & $1.81(0.38-8.49)$ & \\
\hline Tumor stage & & 0.000 & & 0.000 \\
\hline I (36) & 1.0 & & 1.0 & \\
\hline II (26) & $1.13(0.44-2.95)$ & 0.798 & $0.93(0.36-2.44)$ & 0.885 \\
\hline III (24) & $2.94(1.27-6.79)$ & 0.012 & $1.96(0.86-4.71)$ & 0.136 \\
\hline IV (20) & $14.00(6.07-32.28)$ & 0.000 & $14.22(5.79-34.87)$ & 0.000 \\
\hline Mucin phenotype ${ }^{b}$ & & 0.023 & & 0.049 \\
\hline Intestinal (37) & 1.0 & & 1.0 & \\
\hline Non-intestinal (69) & $2.03(1.06-3.89)$ & & $2.04(1.00-4.01)$ & \\
\hline
\end{tabular}

${ }^{\mathrm{a}}$ Adjusted for other variables in the table; ${ }^{\mathrm{b}}$ According to all mucin phenotypes, $\mathrm{p}=0.089$ for unadjusted analysis and $\mathrm{p}=0.088$ for adjusted analysis.

The mucin phenotype correlated well with histologic differentiation $(\mathrm{p}=0.000)$ and Lauren's classification of tumors $(\mathrm{p}=0.003)$. However, the mucin phenotype of tumors did not accord completely with histologic differentiation or Lauren's classification. Among 40 well-differentiated adenocarcinomas, $24(60.0 \%)$ expressed the I-phenotype while only seven (17.5\%), 4 (10.0\%) and $5(12.5 \%)$ expressed the G-, M- and U-phenotypes, respectively. Among 66 poorly differentiated adenocarcinomas, 28 (42.4\%) expressed the
G-phenotype while 13 (19.7\%), 18 (27.3\%) and 7 (10.6\%) expressed the I-, M- and U-phenotypes, respectively. Twenty-eight out of $35(80.0 \%)$ G-phenotype cancers were poorly differentiated and 24 out of 37 (64.9\%) I-phenotype cancers were well-differentiated (Table III). Lauren's intestinal type cancers accounted for $64.9 \%$ (24/37) of Iphenotype cancers while Lauren's diffuse type accounted for $71.4 \%(25 / 35)$ of G-phenotype cancers. I-phenotype accounted for $54.5 \%(24 / 44)$ of Lauren's intestinal type 
cancers while G-phenotype accounted for $40.3 \%$ (25/62) of Lauren's diffuse type cancers (Table II).

According to the expression of each mucin, the expression of Con A correlated well with histologic differentiation $(\mathrm{p}=0.038)$, but there was no significant correlation between the expression of other mucins and the other patient clinicopathologic variables. Among 28 Con A-expressing tumors, $22(78.6 \%)$ were poorly-differentiated type cancers, but only a third of poorly-differentiated tumors expressed Con A. The expression of CD10 was less frequent in the poorly-differentiated than well-differentiated type without statistical significance $(\mathrm{p}=0.068)$. The expression of Con A tended to be related with tumor location. Its expression rate was higher in the tumors of cardia/body compared to antral tumors $(\mathrm{p}=0.084$ ) (data not shown).

Intestinal phenotype cancers show a higher survival rate than non-intestinal phenotypes. For $\sim 10$ years, the overall survival rate of 106 patients with gastric adenocarcinomas was $51.9 \%$ (Fig. 2A). Unadjusted analyses revealed no significant difference in survival rates according to patients' age $(\mathrm{p}=0.299)$ and gender $(\mathrm{p}=0.171)$, and mucin phenotype $(\mathrm{p}=0.089$, Fig. 2E). Histologic differentiation, Lauren's classification and the tumor stage were associated with survival rate $(\mathrm{p}=0.016, \mathrm{p}=0.011$ and $\mathrm{p}=0.000$, Fig. $2 \mathrm{~B}-\mathrm{D}$ and Table III) on univariate analysis. When survival rate was adjusted for clinical and histopathologic variables, the tumor stage and patients' age were associated with survival rate ( $\mathrm{p}=0.000$ and $\mathrm{p}=0.001$, respectively, Table III). Histologic differentiation and Lauren's classification were had no prognostic significance on multivariate analysis $(\mathrm{p}=0.975$ and $\mathrm{p}=0.454$, respectively, Table III). However, the mucin phenotypes showed a difference in survival $(\mathrm{p}=0.088)$ and the I-phenotype cancers showed a better outcome than Gphenotypes (HR 0.47, 95\% CI 0.21-1.03, p=0.059, data not shown) on adjusted analysis, although there was no statistical significance. Moreover, when the tumors were reclassified as I- and non I-phenotypes, the non I-phenotype cancers showed a poorer outcome than I-phenotypes on unadjusted (HR 2.03, 95\% CI 1.06-3.89, $\mathrm{p}=0.023$ ) and adjusted analyses (HR 2.04, $95 \%$ CI $1.00-4.01, p=0.049$, Table III). According to the expression of each mucin, no significant difference in survival rate was found on unadjusted and adjusted analyses (data not shown).

\section{Discussion}

The present study is the longest follow-up study, conducted to date, evaluating the prognostic significance of mucin phenotype of gastric adenocarcinoma. We followed 106 patients with gastric cancers for $\sim 10$ years after curative resection, and found that the expression of I-phenotype was an independent good prognostic factor together with tumor stage and patients' age while histologic differentiation and Lauren's classification did not show prognostic significance on multivariate analysis.

When the mucin phenotype of gastric adenocarcinoma correlated with various clinicopathologic factors, it related well with the histologic differentiation $(p=0.000)$ and Lauren's classification of tumors $(p=0.003)$ which have been commonly used as prognostic factors. Over half $(60.0 \%)$ of the well-differentiated cancers expressed the I-phenotype and $42.4 \%$ of poorly differentiated cancers expressed the Gphenotype. Furthermore, the majority (80.0\%) of G-phenotype cancers was poorly differentiated and $64.9 \%$ of I-phenotype cancers were well-differentiated. These findings are consistent with the previous report (19) that G-phenotype tumors were associated with a higher rate of undifferentiated types as compared to I-phenotype tumors $(p<0.05)$. Sixtyfive percent of I-phenotype cancers were Lauren's intestinal type and $71 \%$ of G-phenotype cancers were Lauren's diffuse type. However, despite a well-defined correlation between the mucin phenotype and histologic classification, they did not concur completely with each other, that is, $40 \%$ of welldifferentiated cancers expressed non I-phenotype and $57.6 \%$ of poorly-differentiated cancers expressed non-G phenotype. Likewise, $45.5 \%$ of Lauren's intestinal type tumors also expressed non-I-phenotypes, and $59.7 \%$ of Lauren's diffuse type tumors also expressed other types than the G-phenotype. These findings suggest that different mechanisms may affect the mucin histogenesis of gastric adenocarcinoma. Therefore, it may be necessary to clarify the significance of such phenotypic expressions and the histogenesis of gastric adenocarcinoma.

Recently, Barresi et al (20) examined 40 gastric adenocarcinomas for MUC1 and MUC2 immunohistochemical expression to verify whether there is a correlation between mucin phenotype and Lauren's classification. Their results were contradictory to ours. They found that the Gphenotype was significantly higher in Lauren's intestinaltype adenocarcinomas, whereas cases showing an Iphenotype were significantly more frequent in Lauren's diffuse-type adenocarcinomas. This obvious discrepancy may come from the difference in the classification of mucin phenotype and the very small number of patients analyzed. They determined the mucin phenotype based on the expression pattern of MUC1 and MUC2. However, the mucin phenotypes were determined according to the expression patterns of CD10, MUC2, HGM and Con A binding in the present study. The mucin expression pattern of gastric carcinomas is heterogeneous (10-14). Therefore, to determine an accurate distribution of mucin phenotypes of gastric adenocarcinomas, it has been suggested that the use of a combination of antigen targets and lectin binding, namely HGM or MUC5AC and MUC6 or Con A staining, would be essential for determining the G-phenotype, and a combination of MUC2 for goblet cells and their precursor cells (high-iron diamine-alcian blue staining is not specific for goblet cells and does not detect goblet cell precursors) and CD10 for enterocytes for determination of the Iphenotype (21).

For follow-up periods of $\sim 10$ years, the overall survival rate was $51.9 \%$. The unadjusted and adjusted analyses among all mucin phenotypes only showed a trend for a difference in outcomes ( $\mathrm{p}=0.089$ and $\mathrm{p}=0.088$, respectively). In addition, I-phenotype cancers tended to show a higher survival rate than G-phenotype tumors without statistical significance on adjusted analysis $(\mathrm{p}=0.059)$. In the present study, therefore, the mucin phenotype of cancers was classified as I-phenotype vs. non-I-phenotype for the survival analysis. The histologic 
differentiation $(\mathrm{p}=0.009)$, Lauren's classification $(\mathrm{p}=0.011)$ and tumor stage $(\mathrm{p}=0.0001)$ had a prognostic significance on univariate analysis. The I-phenotype cancers also showed a better outcome than non-I-phenotypes on unadjusted analysis $(\mathrm{p}=0.032)$. When the survival rate was adjusted for the patients' clinicopathologic variables using Cox hazard models, the analysis showed the I-phenotypic expression of a tumor to be a good independent prognostic factor $(p=0.049)$, together with a lower tumor stage $(p=0.000)$ and patients' younger age $(p=0.002)$. The histologic differentiation $(\mathrm{p}=0.975)$ and Lauren's classification $(\mathrm{p}=0.454)$ had no prognostic significance on adjusted analysis. Recently, both univariate and multivariate analyses of survival rates revealed G-phenotype tumors to be associated with a significantly poorer outcome than Iphenotype tumors $(\mathrm{p}<0.05)(9)$. In addition, Tajima et al (22) reported that the incidence of $\mathrm{G}$-phenotypes were significantly higher in tumors with peritoneal recurrence $(p=0.0002)$, while the incidence of MUC2-negative tumors and CD10-positive tumors were significantly higher in tumors with hematogenous recurrence $(p=0.028$ and $p=0.039$, respectively) than in tumors without recurrence. These findings suggest that the gastric and intestinal phenotypic classification of gastric carcinoma can also be used to predict the pattern of its recurrence after curative resection. The above findings of the present study, in conjunction with previous reports, strongly suggest that the mucin phenotype classification could be useful not only for investigating the tumorigenesis of gastric carcinoma, but also for determining prognosis.

In summary, although the mucin phenotype correlated well with histologic differentiation and Lauren's classification of tumors, it was quite different from those histologic types in many cases. Moreover, the histologic differentiation and Lauren's classification did not have prognostic significance on multivariate analysis, but Iphenotypic expression of a tumor was an independent good prognostic factor, together with a lower tumor stage and the patients' younger age. Therefore, mucin phenotype may have an important role as a prognostic factor of gastric adenocarcinomas, compared to conventional histologic classifications. Further studies with larger sample sizes are required to better assess the clinical significance of mucin phenotypes of gastric adenocarcinoma.

\section{Acknowledgements}

This study was supported by grant award from Gyeongsang National University Hospital (Special clinical fund 2006).

\section{References}

1. Lauren P: The two histological main types of gastric carcinoma: diffuse and so-called intestinal-type carcinoma. An attempt at a histo-clinical classification. Acta Pathol Microbiol Scand 64: $31-49,1965$

2. Nakamura K, Sugano H and Takagi K: Carcinoma of the stomach in incipient phase: its histogenesis and histological appearances. Gann 59: 251-258, 1968

3. Garnier P, Vielh P, Asselain B, et al: Prognostic value of the Lauren and Ming classifications in gastric adenocarcinoma. Multidimensional analysis. Gastroenterol Clin Biol 12: 553-558, 1988.
4. Ribeiro MM, Sarmento JA, Sobrinho Simoes MA and Bastos J: Prognostic significance of Lauren and Ming classifications and other pathologic parameters in gastric carcinoma. Cancer 47: 780-784, 1981.

5. Endoh Y, Sakata K, Tamura G, et al: Cellar phenotypes of differentiated-type adenocarcinomas and precancerous lesions of the stomach are dependent on the genetic pathways. J Pathol 191: 257-263, 2000.

6. Koseki K, Takizawa T, Koike M, Ito M, Nihei Z and Sugihara K: Distinction of differentiated type early gastric carcinoma with gastric type mucin expression. Cancer 89: 724-732, 2000.

7. Tajima Y, Nakanishi Y, Yoshino T, Kokawa A, Kusano M and Shimoda T: Clinicopathological study of early adenocarcinoma of the gastric cardia: comparison with early adenocarcinoma of the distal stomach and esophagus. Oncology 61: 1-9, 2001.

8. Kabashima A, Yao T, Sugimachi K and Tsuneyoshi M: Relationship between biologic behavior and phenotypic expression in intramucosal gastric carcinomas. Hum Pathol 33: 80-86, 2002.

9. Tajima Y, Shimoda T, Nakanishi Y, et al: Gastric and intestinal phenotypic marker expression in gastric carcinomas and their prognostic significance: immunohistochemical analysis of 136 lesions. Oncology 61: 212-220, 2001.

10. Ho SB, Shekels LL, Toribara NW, et al: Mucin gene expression in normal, preneoplastic, and neoplastic human gastric epithelium. Cancer Res 55: 2681-2690, 1995.

11. Filipe MI, Linehan JM, Durrant LG, et al: Expression of a peptide epitope of the colonic mucin MUC2 in precursor lesions to gastric carcinoma. Eur J Cancer Prev 5: 287-295, 1996.

12. Reis CA, Sorensen T, Mandel U, et al: Development and characterization of an antibody directed to an alpha-N-acetyl-Dgalactosamine glycosylated MUC2 peptide. Glycoconj J 15: 51-62, 1998.

13. Correa P: Human gastric carcinogenesis: a multistep and multifactorial process-first American Cancer Society award lecture on cancer epidemiology and prevention. Cancer Res 52: 6735-6740, 1992.

14. Filipe MI, Münoz N, Matko I, et al: Intestinal metaplasia types and the risk of gastric cancer: a cohort study in Slovenia. Int J Cancer 57: 324-329, 1994.

15. Watanabe H, Jass JR and Sobin LH: Histological typing of esophageal and gastric tumors. WHO International histological classification of tumours. Springer-Verlag, Berlin, 1990.

16. Katsuyama $T$ and Spicer SS: Histochemical differentiation of complex carbohydrates with variants of the concanavalin Ahorseradish peroxidase method. J Histochem Cytochem 26: 233-250, 1978 .

17. Ohmura K, Tamura G, Endoh Y, Sakata K, Takahashi T and Motoyama T: Microsatellite alterations in differentiated-type adenocarcinomas and precancerous lesions of the stomach with special reference to cellular phenotype. Hum Pathol 31: 1031-1035, 2000.

18. Shiroshita H, Watanabe H, Ajioka Y, Watanabe G, Nishikura K and Kitano S: Re-evaluation of mucin phenotypes of gastric minute well differentiated-type adenocarcinomas using a series of HGM, MUC5AC, MUC6, M-GGMC, MUC2 and CD10 stains. Pathol Int 54: 311-321, 2004.

19. Williams SJ, Wreschner DH, Tran M, Eyre HJ, Sutherland GR and McGuckin MA: MUC13, a novel human cell surface mucin expressed by epithelial and hemopoietic cells. J Biol Chem 276: 18327-18336, 2001.

20. Barresi V, Vitarelli E, Grosso M, Tuccari G and Barresi G: Relationship between immunoexpression of mucin peptide cores MUC1 and MUC2 and Lauren's histologic subtypes of gastric carcinomas. Eur J Histochem 50: 301-309, 2006.

21. Watanabe G, Watanabe H, Ajioka Y, Shiroshita H and Nishikura K. Well-differentiated type adenocarcinomas of gastric mucin phenotype transform into intestinal type carcinomas. I to Cho (Stomach Intestine) 38: 693-700, 2003 (In Japanese with English summary).

22. Tajima Y, Yamazaki K and Nishino N, et al: Gastric and intestinal phenotypic marker expression in gastric carcinomas and recurrence pattern after surgery-immunohistochemical analysis of 213 lesions. Br J Cancer 91: 1342-1348, 2004. 